\title{
Hydrogen Gas Sensor Based on ZnO Nanoroads Grown on Si by Thermal Evaporation
}

\author{
Hind Ibraheem Abdulgafour ${ }^{1}$, Hassan Hadi Darwoysh ${ }^{1,2}$ and Faez Mohamad Hassan ${ }^{2 *}$ \\ 1. Departement of Physics, Ministry of Science and Technology, Baghdad, AlKerrada 10069, Iraq \\ 2. Department of Physics, College of Education, Al-Mustansiriya University, Baghdad, New Baghdad 10062, Iraq
}

\begin{abstract}
High-quality $\mathrm{ZnO}$ (Zinc oxide) nanorods grown on Si substrate have been synthesized for hydrogen gas sensor application through a low-cost catalyst-free process by thermal evaporation at $800{ }^{\circ} \mathrm{C}$. The morphological, structural and optical properties of the $\mathrm{ZnO}$ nanorods have been examined. In this study, $\mathrm{Pd} / \mathrm{ZnO} / \mathrm{Pd} \mathrm{MSM}$ (Metal-semiconductor-metal) gas sensor has been fabricated based on the $\mathrm{ZnO}$ nanorods. The absence of a seed layer and the coalescence of $\mathrm{ZnO}$ nanorods were the key factors responsible for the high sensitivity of the gas sensor at room temperature. The sensitivity of $\mathrm{ZnO}$ nanorods is measured at different concentrations from $25 \mathrm{ppm}$ to $150 \mathrm{ppm}$ of $\mathrm{H}_{2}$ gas at room temperature. The highest response of the $\mathrm{ZnO} / \mathrm{Si}$ sensor was $110 \%$ in the presence of 500 ppm of $\mathrm{H}_{2}$. This high sensitivity can be attributed to the high surface-area- to-volume ratio of the nanorods between the Pd contacts of the MSM configuration.
\end{abstract}

Key words: $\mathrm{ZnO}$, nanostructures, thermal evaporation, gas sensor.

\section{Introduction}

Leaked hydrogen fuel could have small negative effects on atmosphere. Using hydrogen as an energy carrier can help reduce air pollution and GHG (Greenhouse gas) emissions associated with fossil fuels. However, if used on a large-scale, it is important that hydrogen does not leak significantly into the atmosphere as it might have some negative environmental effects, such as increasing the lifetime of methane, increasing climate effects and causing some depletion of the ozone layer [1]. In recent years, low-dimensional systems have attracted tremendous interest in nanosensor applications due to their gas sensitivity, UV (Ultraviolet) photoresponse, and optical transparency in the visible region, among other features $[2,3]$. In particular, quasi-one dimensional nanowires or nanorods are promising low-cost material for high-speed UV photoconductive nanoscale detectors and gas sensors [4-6]. In the past decade, research on wide band gap semiconductors

\footnotetext{
*Corresponding author: Faez Mohamad Hassan, Ph.D.,
} research fileds: solid state physics and nanotechnology. has focused on $\mathrm{ZnO}$ (zinc oxide) due to its excellent properties as a semiconductor. Its high electron mobility, high thermal conductivity, good transparency, wide and direct band gap $(3.37 \mathrm{eV})$, large exciton binding energy and ease of fabrication into micro- and nanostructures make $\mathrm{ZnO}$ suitable for a wide range of applications in optoelectronics, transparent electronics, lasing and sensing [7, 8].

Nanorods are some of the most common forms of $\mathrm{ZnO}$ that possess several excellent properties, such as high sensitivity to adsorbed oxygen on the surface, excellent electric transport, high photo-sensitivity, optical wave-guiding, and large surface area-to-volume ratio [9]. Compared with the bulk materials used in gas sensors, such as thin films, nanorods have greater gas sensitivity and selectivity and lower operating temperatures $[10,11]$. Room-temperature hydrogen gas sensors have received a great deal of interest for use in several applications because of the extremely low power consumption, ability to be used safely in flammable environments, and long lifetime [12-14]. For gas sensing applications, $\mathrm{ZnO}$ is one of the promising 
metal oxide wide band gap semiconductors [16]. In gas sensing, $\mathrm{ZnO}$ has been tested for sensing harmful and toxic gases [15]. A common concern about gas sensors based on $\mathrm{ZnO}$ thin films is the lack of selectivity and higher operating temperature. In general, its optimum operating temperature range is 400-450 ${ }^{\circ} \mathrm{C}$ [16]. Also, Ref. [17] used Pt-coated $\mathrm{ZnO}$ nanorods as sensors that were capable of detecting part per million (ppm) concentrations of hydrogen at room temperature. According to the previous reports, the sensitivity of $\mathrm{ZnO}$ to gas may be affected by nanostructure surface defects and post-growth annealing in $\mathrm{H}_{2}$ or $\mathrm{O}_{2}$ ambient [18]. In this study, an alternative method to synthesize high quality $\mathrm{ZnO}$ nanorods on $\mathrm{SiO}_{2} / \mathrm{Si}$ by using thermal evaporation method without catalyst is presented. The simplicity, lower cost and suitability of this method in producing high structural and optical quality $\mathrm{ZnO}$ nanostructures are very promising for efficient sensing applications; Particularly, the high surface area to volume ratio is the major factor responsible for high performance of the sensors fabricated based on $\mathrm{ZnO}$ nanorods.

\section{Matreials and Methods}

\subsection{Synthesis of ZnO Nanorods}

The synthesis process was carried out in a controllable tube furnace with a quartz tube (inner diameter, $25 \mathrm{~mm}$ ). N-type silicon ( $\mathrm{Si}$ ) wafers cut into $1 \times 1 \mathrm{~cm}^{2}$ pieces with a single polished side were used as substrates. The substrates were ultrasonically cleaned with acetone and IPA (Isopropyl alcohol), and then rinsed with DI water. A pure metallic $\mathrm{Zn}$ powder $(99.9 \%)$ as source material was placed into a ceramic boat. The Si substrates (with the polished side facing the source material) were inserted into a quartz tube. The furnace was slowly heated from $400{ }^{\circ} \mathrm{C}$ to $800{ }^{\circ} \mathrm{C}$. After reaching $800{ }^{\circ} \mathrm{C}$, argon (Ar) was introduced with flow rates of 350 scrums, respectively, for 1 hour. After the evaporation, the alumina boat was slowly drawn out from the furnace and cooled to room temperature. A white layer of $\mathrm{ZnO}$ was observed on the Si substrate surface. The thickness of $\mathrm{ZnO}$ thin film was around $200 \mathrm{~nm}$ was measured by reflectometer (F20). The surface morphology and structure of the $\mathrm{ZnO}$ nanostructures were examined by SEM (Scanning electron microscopy) JEOL, attached with an energy dispersive X-ray spectroscopy (EDS). The HR-XRD (High resolution X-ray diffractometer) was used for phase identifications.

\subsection{Fabrication of Gas Sensor}

The (I-V) measurements of the sample were carried out using the Keithley model 2400. Fig. 1 shows the schematic diagram of the gas sensing system. To fabricate a diode gas sensor, MSM with interdigitated contacts (electrodes) forming Schottky barriers was used.

The four fingers of each of the Pd contact electrodes were $0.23 \mathrm{~mm}$ wide and $4 \mathrm{~mm}$ long, with $0.4 \mathrm{~mm}$ spacing, as shown in Fig. 2. The gas sensing experiments were carried out by using a homemade gas sensing chamber. For characterization of the gas sensing device, the test fixture was placed in the chamber with wires connected from the probes to Keithley device to measure the current voltage (I-V) characteristics of the sample. The voltage was biased from $5 \mathrm{~V}$ to $-5 \mathrm{~V}$ and different concentrations of $2 \%$ $\mathrm{H}_{2}$ in $\mathrm{N}_{2}$ gas were used in this experiment.

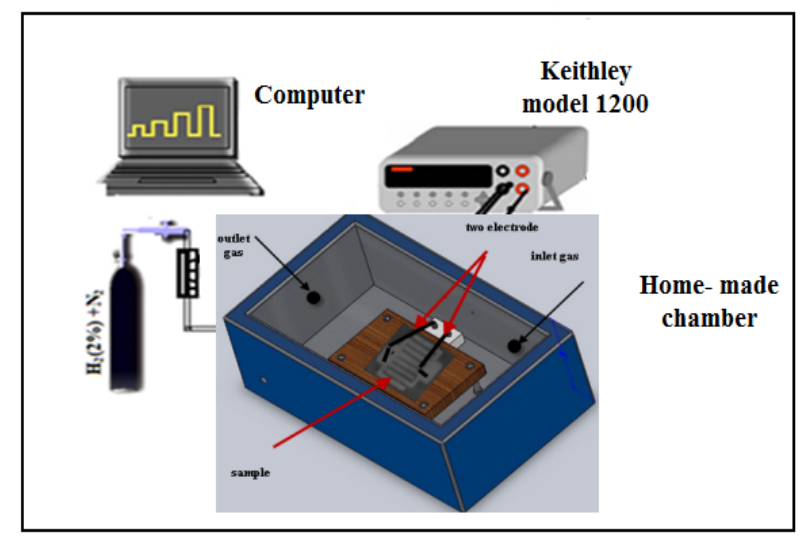

Fig. 1 Schematic diagram of the home-made gas sensor system. 


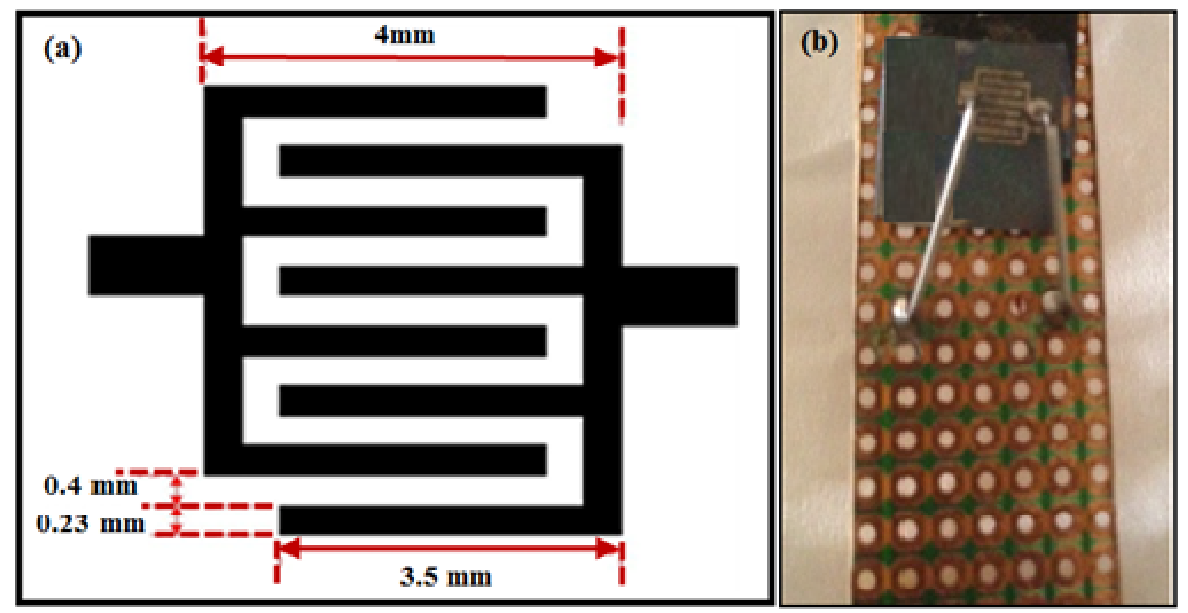

Fig. 2 (a) The structure used in the fabrication of MSM; (b) The photo image MSM gas sensor fabricated on ZnO nanorods.

\section{Results and Discussion}

\subsection{Characterization of the $\mathrm{ZnO}$ Nanorods}

The SEM image is shown in Fig. 3. The resulting images of the prepared $\mathrm{ZnO}$ depict nanorods structure. The nanorods were linked with neighboring nanorods, which formed a nanorod network. The structures of $\mathrm{ZnO}$ nanorod morphology revealed a hexagonal structure with average diameters are about 80-100 nm, and their lengths were more than $500 \mathrm{~nm}$ with thickness around $200 \mathrm{~nm}$ (Fig. 3).

The XRD pattern shows the hexagonal (wurtzite) structure of the $\mathrm{ZnO}$ nanorods. Strong diffraction intensity peaks related to the (002) plane of the $\mathrm{ZnO}$ nanorods were observed (Fig. 4). The strongest peak (002) at $2 \theta=34.37^{\circ}$ with FWHM (Full-width half-maximum) of $0.19^{\circ}$ showed an excellent quality of the $\mathrm{ZnO}$ nanorods that the preferred growth orientation of nanorods is along the c-axis. Furthermore, the strong intensity and narrow width of the $\mathrm{ZnO}$ diffraction peaks also indicate that the resulting products had well crystalline.

\subsection{Sensing Characterization}

These nanorods provide a large surface-area-to-volume ratio to interact with the surrounding gas. The change in the depletion diameter fully occurs in the $\mathrm{ZnO}$ nanorods, which result in a significant change in conductivity. In ambient air, the $\mathrm{ZnO}$ nanorod surface adsorbs oxygen $\left(\mathrm{O}_{2}\right)$ molecules,

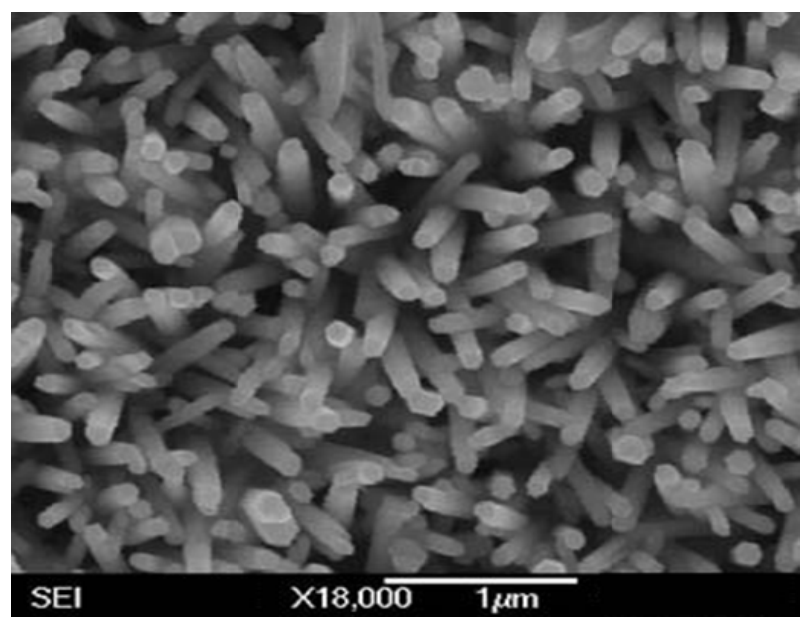

Fig. 3 The SEM images of $\mathrm{ZnO}$ nanorods grown at $800{ }^{\circ} \mathrm{C}$.

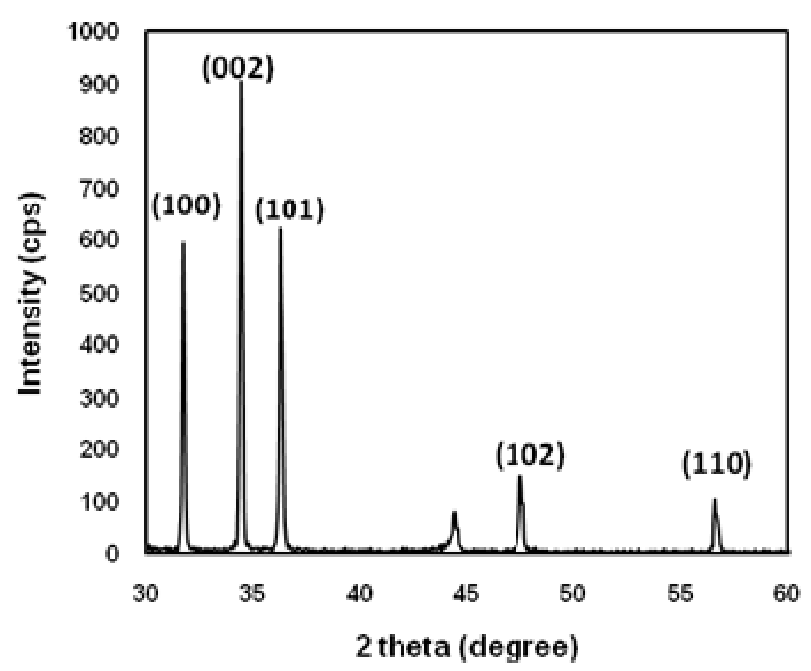

Fig. 4 The XRD spectra of $\mathrm{ZnO}$ grown at $850{ }^{\circ} \mathrm{C}$. 
which results in increased resistivity. In this work, the $\mathrm{Pd} / \mathrm{ZnO} / \mathrm{Pd}$ gas sensor has been successfully fabricated using high-quality $\mathrm{ZnO}$ nanorods. Fig. 5 shows the I-V characteristics (linear scale plot) of the $\mathrm{Pd} / \mathrm{ZnO}$ nanorods hydrogen gas sensors measured for different concentrations $(25,50,75,100,125$, and 150 ppm) of $\mathrm{H}_{2}$ gas at room temperature $(300 \mathrm{~K})$. The sensor was found to show good behavior with remarkable increasing of current at different concentrations of $\mathrm{H}_{2}$ gas with bias voltage from $5 \mathrm{~V}$ to - $5 \mathrm{~V}$ for $\mathrm{ZnO}$ deposited on $\mathrm{Si}$ substrate. The increment of the current in the presence of hydrogen may be due to a decrease in the barrier height between the $\mathrm{Pd} / \mathrm{ZnO}$ interfaces after $\mathrm{H}_{2}$ adsorption.

The sensitivity $(S)$ was determined as the ratio $\left(\Delta R_{\text {gas }} / R_{\text {air }}\right)$ of the resistance in hydrogen gas $\left(R_{\text {gas }}\right)$ to that in air $\left(R_{\text {air }}\right)$ or also could be measured as a reference to the current $\left(\Delta I_{g a s} / I_{a i r}\right)$ of the current in hydrogen gas $\left(I_{\text {gas }}\right)$ to current in air $\left(I_{\text {air }}\right)$, at fixed applied voltage $(1 \mathrm{~V})$. Fig. 6 shows the variation of sensitivity with the $\mathrm{H}_{2}$ different concentrations at room temperature for $\mathrm{Pd} / \mathrm{ZnO}$ recorded at $1 \mathrm{~V}$ in forward current mode. One may observe that the sensitivity increased gradually with the hydrogen flow rate for this device.

From Fig. 6, it could be seen the sensitivity at high concentration of hydrogen gas (150 ppm) was around $110 \%$ for $\mathrm{ZnO}$ nanorods at room temperature; this could be due to the improvement of the structural properties of the $\mathrm{ZnO}$ nanorods. On the other hand, this might also be due to the increase of the internal surface area, which allows the enhancement of the adsorbate effects; and high activity in surface chemical reactions [19]. Furthermore, this observation coincides with the fact that $\mathrm{ZnO} / \mathrm{Si}$ sample have a large specific area and therefore provide a large contact area, hence giving it a higher chance to react with gases and increase the sensitivity. The series resistances evaluated from the $\mathrm{I}-\mathrm{V}$ curves for $\mathrm{Pd} / \mathrm{ZnO}$ Schottky diode measured at $300 \mathrm{~K}$ with different concentrations of $\mathrm{H} 2$ gas are shown in Fig. 7. The series resistance decreased exponentially upon the introduction of different concentrations of $2 \% \mathrm{H}_{2}$ in $\mathrm{N}_{2}$ gas for the $\mathrm{ZnO}$ nanorods.

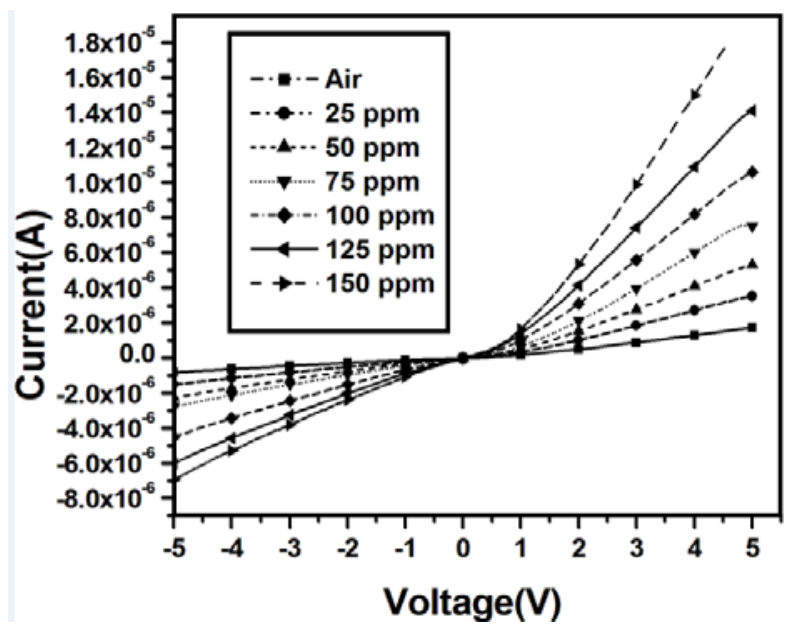

Fig. 5 I-V characteristics (linear scale plot) of Pd/ZnO measured at room temperature for different hydrogen concentrations.

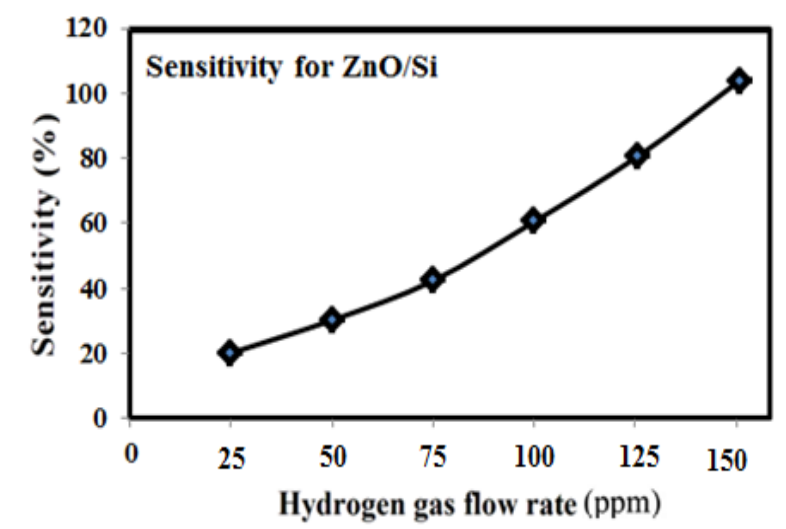

Fig. 6 The sensitivity of Pd/ZnO for nanorods grown on Si substrate.

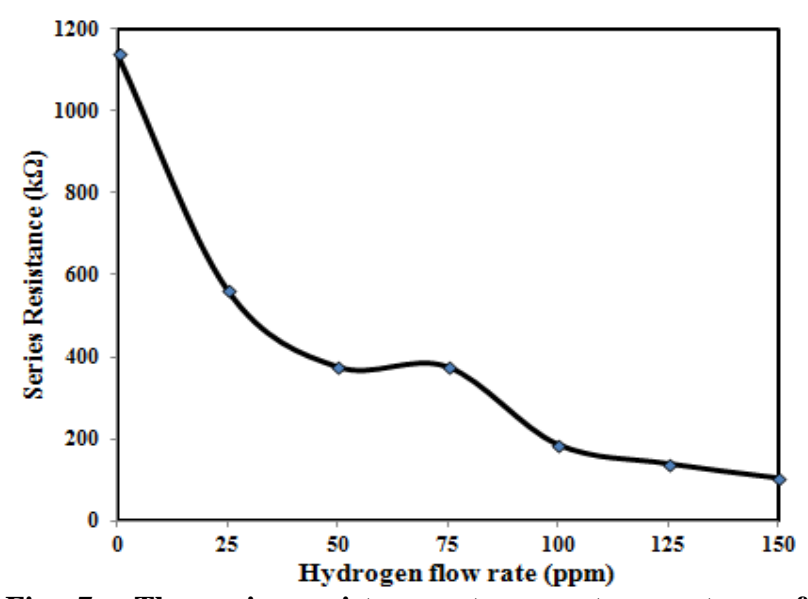

Fig. 7 The series resistance at room temperature of $\mathrm{Pd} / \mathrm{ZnO}$ nanorods as a function of hydrogen concentrations. 


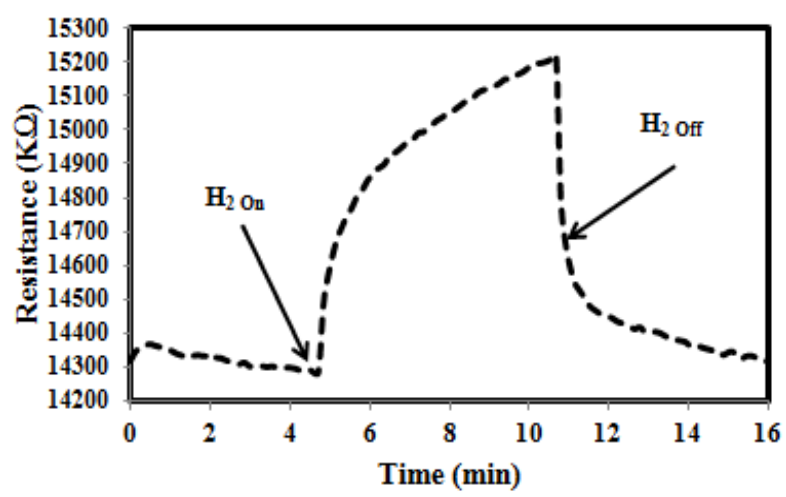

Fig. 8 The response time behavior of $\mathrm{Pd} / \mathrm{ZnO}$ nanorods Schottky diode sensor at $1 \mathrm{~V}$.

A part from that, the response time is defined as the time taken for the sensor to reach $90 \%$ of the saturation current from initial value while the recovery time is defined as the time taken from saturation current to reach $10 \%$ of its saturation current value after contact of the test gas with the surface of the sensor. Measured resistance at a bias of $1 \mathrm{~V}$ as a function of time is shown in Fig. 8 for $\mathrm{Pd} / \mathrm{ZnO}$ exposed to $2 \% \mathrm{H}_{2}$ in $\mathrm{N}_{2}$ in constant concentration 150 ppm at room temperature. As can be clearly seen from Fig. 8, the adsorption and desorption times for $\mathrm{H}_{2}$ were set at $10 \mathrm{~min}\left(\mathrm{H}_{2}\right.$ on $)$ and $15 \mathrm{~min}\left(\mathrm{H}_{2}\right.$ off $)$, respectively. The response and recovery times measured for the sensor were about 5 and $6 \mathrm{~min}$, respectively. Fig. 8 shows the analysis/plot of the rise time and recovery time as a function of $\mathrm{H}_{2}$ gas concentration (150 ppm). Also, after the injection of hydrogen into the measuring chamber, the resistance of samples changes and becomes stable. After the removal of hydrogen, the resistance of samples reverts to values before hydrogen injection and becomes stable again. It may be seen that the maximum resistance could be attained in less than $10 \mathrm{~min}$. The experimental results indicate the potential of using zinc oxide nanorods face for gas sensing based on Schottky Barriers of $\mathrm{Pd} / \mathrm{ZnO}$. Compared with conventional $\mathrm{ZnO}$ thin films, the observed enhancement in the gas sensing properties of the $\mathrm{ZnO}$ nanorods gas sensor is most likely attributable to the relatively higher degree of surface reaction due to the high specific surface area associated with the nanostructure. Also, a comparison of $\mathrm{ZnO}$ rods-like nanostructures with other nanostructures leads us to conclude that the gas sensing properties also depend on other factors, including crystallinity and surface properties, and these need to be studied further.

\section{Conclusions}

In summary, the growth of $\mathrm{ZnO}$ rods-like nanostructure arrays on $\mathrm{Si}$ substrates has been demonstrated at $800{ }^{\circ} \mathrm{C}$. The hydrogen gas sensor has been fabricated based on $\mathrm{ZnO}$ nanorods synthesized by a catalyst-free thermal evaporation technique by simple and low-cost process worked at room temperature. The good response of the $\mathrm{ZnO}$ nanorods gas sensor was $110 \%$ when $150 \mathrm{ppm}$ of $\mathrm{H}_{2}$ gas was injected. The series resistance in the $\mathrm{Pd} / \mathrm{ZnO}$ nanostructures was decreased exponentially with increasing the flow rate of $\mathrm{H}_{2}$ gas. The enhanced sensitivity at a lower operating temperature for the $\mathrm{ZnO}$ nanorods is attributed to the reduction of grain size and increase in the surface area. As a consequence, the surface activity is enhanced, and the activation energy of the surface-chemisorbed $\mathrm{H}_{2}$ gases is reduced, enhancing the $\mathrm{H}_{2}$ gas adsorption.

\section{References}

[1] Van Ruijven, B., Lamarque J.-F. and Van Vuuren, D. P. 2011. "Emission Scenarios for a Global Hydrogen Economy and the Consequences for Global Air Pollution." Global Environmental Change 21: 983-94.

[2] Heo, Y. W., Norton, D. P., Tien, L. C., Kwon, Y., Kang, B. S. and Ren, F. et al. 2004. "ZnO Nanowire Growth and Devices." Materials Science and Engineering: R: Reports 7: 1-47.

[3] Li, Q. H., Liang, Y. X., Wan, Q. and Wang, T. H. 2004. "Oxygen Sensing Characteristics of Individual $\mathrm{ZnO}$ Nanowire Transistors." Applied Physics Letters 85: 6389-91.

[4] Chow, L., Lupan, O., Heinrich, H. and Chai, G. 2009. "Self-Assembly of Densely Packed and Aligned Bilayer ZnO Nanorod Arrays." Applied Physics Letter 94: 163105-8.

[5] Lupan, O., Chai, G. and Chow, L. 2007. "Fabrication of ZnO Nanorod-Based Hydrogen Gas Nanosensor." 
Microelectronics Journal 38: 1211-6.

[6] Chai, G., Lupan, O., Chow, L. and Heinrich, H. 2009. "Crossed Zinc Oxide Nanorods for Ultraviolet Radiation Detection." Sensors Actuators A Physics 150: 184-7.

[7] Bai, X. D., Gao, P. X., Wang, Z. L. and Wang, E. G. 2003. "Measuring the Work Function at a Nanobelt Tip and at a Nanoparticle Surface." Applied Physics Letters 82: 4806-8.

[8] Janotti, A. and Van de Walle, C. G. 2009. "Fundamentals of Zinc Oxide as a Semiconductor." Reports on Progress in Physics 72: 126501-9.

[9] Akhavan, O., Mehrabian, M., Mirabbaszadeh, K. and Azimirad, R. 2009. "Hydrothermal Synthesis of $\mathrm{ZnO}$ Nanorod Arrays for Photocatalytic Inactivation of Bacteria." Journal of Physics D: Applied Physics 42: 225305-14.

[10] Schmidt-Mende, L. and MacManus-Driscoll, J. L. 2007. "ZnO-Nanostructures, Defects and Devices." Materials Today 10: 40-84.

[11] Tonezzer, M. and Lacerda, R. G. 2012. "Zinc Oxide Nanowires on Carbon Microfiber as Flexible Gas Sensor." Physica E: Low-Dimensional Systems and Nanostructures 44: 1098-102.

[12] Wright, J. S., Lim, W., Norton, D. P., Pearton, S. J., Ren, F. and Johnson, J. L. et al. 2010. "Nitride and Oxide Semiconductor Nanostructured Hydrogen Gas Sensors."
Semiconductor Science and Technology 25: 024002.

[13] Sun, J., Xu, J., Yu, Y. S., Sun, P. and Lu, F. G. 2012. "UV-Activated Room Temperature Metal Oxide Based Gas Sensor Attached with Reflector." Sensors and Actuators B: Chemical 169: 291-6.

[14] Choi, K. J. and Jang, H. W. 2010. "One-Dimensional Oxide Nanostructures as Gas-Sensing Materials: Review and Issues." Sensors 10 (4): 4083-99.

[15] Morkoc, H. and Ozgur, U. 2008. Zinc Oxide: Fundamentals, Materials and Device Technology. John Wiley \& Sons, Germany.

[16] Lee, J. H. 2009. "Gas Sensors Using Hierarchical and Hollow Oxide Nanostructures: Overview." Sensors and Actuators B: Chemical 140: 319-36.

[17] Tien, L. C., Sadik, P. W., Norton, D. P., Voss, L. F., Pearton, S. J. and Wang, H. T. et al. 2005. "Hydrogen Sensing at Room Temperature with Pt-Coated ZnO Thin Films and Nanorods". Applied Physics Letters 87 (22): 222106.

[18] Ahn, M. W., Park, K. S., Heo, J. H., Park, J. G., Kim, D. W. and Choi, K. J. et al. 2008. "Gas Sensing Properties of Defect-Controlled ZnO-Nanowire Gas Sensor." Applied physics letters 93 (26): 263103.

[19] Korotcenkov G. and Cho, B. K. 2010. "Silicon Personification: State of the Art." Critical Reviews in Solid State and Materials Sciences 35: 153-260. 